\title{
Denervação capsular percutânea no tratamento da displasia coxofemoral canina
}

\author{
Percutaneous hip denervation for the treatment of canine hip dysplasia
}

André Luis Selmi ${ }^{\mathrm{I}, \mathrm{II}}$ Bianca Mota Penteado ${ }^{\mathrm{II}}$ Bruno Testoni Lins ${ }^{\mathrm{II}}$

\section{RESUMO}

A displasia coxofemoral canina (DCF) é uma afecção ortopédica freqüente, comumente dolorosa, decorrente de instabilidade e que leva à doença articular degenerativa. Várias formas de tratamento cirúrgico são descritas, entre elas a denervação capsular a céu aberto. Neste trabalho, é descrita a denervação capsular percutânea e seus efeitos em 92 cães com sensibilidade dolorosa na articulação do quadril atribuída à DCF. Foram avaliados os seguintes aspectos: o grau de claudicação (CLAUD), a dor por escala analógica visual, a perimetria da coxa (PC) e os graus máximos de extensão (GME) e flexão (GMF) passiva do quadril imediatamente antes da cirurgia e aos sete, 15, 30, 180 e 360 dias de pós-operatório (p.o.). Os animais foram pré-medicados e submetidos à anestesia geral inalatória e, posteriormente, a borda acetabular crânio-dorsal foi denervada por meio da introdução percutânea de um pino de Steinman com aproximadamente $4,5 \mathrm{~mm}$ de diâmetro. A CLAUD e a dor foram analisadas por meio da ANOVA e do teste de Friedman. A PC, a GME e a GMF foram analisadas por meio da ANOVA e do teste de Tukey. Foi observada melhora significativa na CLAUD e dor a partir dos 15 dias de p.o. A GME apresentou aumento significativo já aos 30 dias de p.o., enquanto que a PC apresentou aumento significativo após os 60 dias de p.o. Conclui-se que a denervação capsular percutânea é alternativa cirúrgica eficaz em restabelecer a movimentação do quadril displásico e promove melhora significativa da dor e claudicação.

Palavras-chave: denervação capsular percutânea, displasia coxofemoral, cães.

\section{ABSTRACT}

Canine hip dysplasia is a common painfull orthopedic disorder, resulting from articular instability and causing degenerative joint disease. Several treatment options are available, including capsular denervation. This study describes the results of percutaneous capsular denervation in 92 dogs diagnosed with hip dysplasia. Lameness (LAM) and pain on palpation (PN) were determined using a visual analogue scale (VAS), muscle girth (MG) and maximum degree of passive extension (PME) and flexion (PMF) of the hip were determined pre-operatively and at days 7, 15, 30, 180 and 360 postoperatively. Dogs were anesthetized and $4.5 \mathrm{~mm}$ Steinman pin was used to percutaneously scrap the craniodorsal border of the acetabulum. LAM and PN were analyzed by means of ANOVA followed by a Friedman test, whereas MG, PME and PMF were analyzed by means of ANOVA followed by a Tukey test. There was a significant decrease in LAM and PN 15 days following surgery. PME presented a significant increase after 30 days of surgery. It is concluded that the percutaneous hip denervation is an effective method in restoring function in the dysplastic hip.

Key words: percutaneous capsular denervation, hip dysplasia, dogs.

\section{INTRODUÇÃO}

A displasia coxofemoral (DCF) é uma afecção ortopédica freqüente, comumente dolorosa, caracterizada pelo desenvolvimento anormal da articulação coxofemoral com conseqüente deterioração das estruturas articulares (HULSE \& JOHNSON, 2002; RAWSON et al., 2005).

As causas são multifatoriais, incluindo fatores hereditários, ambientais, nutricionais e hormonais, entre outros (HULSE \& JOHNSON, 2002; DASSLER, 2003). Na maioria dos casos, cães com DCF

IPrograma de Pós-graduação em Cirurgia e Anestesiologia Veterinária, Universidade de Franca (UNIFRAN), Franca, SP, Brasil. Email: andre_selmi@yahoo.com.br. Autor para correspondência.

IICurso de Medicina Veterinária, Universidade Anhembi Morumbi, São Paulo, SP, Brasil. 
são apresentados para avaliação quando os sinais clínicos se tornam óbvios para o proprietário, o que pode ocorrer antes dos três meses de idade. O histórico e os sinais clínicos geralmente incluem anormalidades no andar, tais como claudicação, passos curtos, salto igual a coelho, assim como diminuição na tolerância ao exercício e dificuldade em levantar-se e subir degraus (TOMLINSON \& McLAUGHLIN, 1996; HULSE \& JOHNSON, 2002; RAWSON et al., 2005).

Entre as modalidades de tratamento, são descritas a conservativa e a cirúrgica. O tratamento conservativo da DCF inclui recomendações nutricionais, controle de peso, restrição a exercícios, reabilitação física, tratamento da dor e administração de suplementos nutracêuticos (TOMLINSON \& McLAUGHLIN, 1996). Já as opções cirúrgicas incluem sinfisiodese púbica juvenil (DUELAND et al. 2001), ressecção da cabeça e do colo femorais (RAWSON et al., 2005), osteotomia pélvica tripla (SCHULZ e DEJARDIN, 2003), substituição total do quadril (OLMSTEAD et al., 1983) e denervação capsular (DC) (GASSE et al., 1996; KINZEL et al., 2002a,b; BRAUN et al., 2003; FERRIGNO et al., 2004, FERRIGNO et al., 2007). Esta última técnica se baseia na denervação da cápsula articular coxofemoral, obtida pela destruição dos ramos articulares do nervo glúteo cranial e ciático, por meio da desperiostização da borda acetabular craniolateral (GASSE et al., 1996; KINZEL et al., 1998). Recentemente, SCHMAEDECKE (2004) descreveu a existência de fibras nervosas em toda a extensão do periósteo acetabular, mostrando maior densidade de fibras na região craniolateral e dorsal do que na região caudolateral do acetábulo.

A gama de abordagens disponíveis no tratamento da DCF destina-se a pacientes com alterações específicas do quadril e as opções de tratamento nos cães com doença articular degenerativa (DAD) se resumem principalmente à ressecção da cabeça e do colo de fêmur e à DC descrita por KINZEL et al. (1998) e posteriormente modificada por FERRIGNO et al. (2004). O objetivo deste estudo foi realizar a avaliação clínica da denervação capsular percutânea (DCP) à articulação coxofemoral canina.

\section{MATERIAL E MÉTODOS}

Foram utilizados 92 cães com diagnóstico clínico de displasia coxofemoral, uni ou bilateral, sintomáticos, sem quaisquer outras alterações no sistema musculoesquelético. Os cães apresentaram confirmação radiográfica da doença, evidenciada pela presença de DAD. Não foram realizadas restrições referentes a gênero, à raça, ao peso ou à idade para inclusão neste estudo, entretanto, aqueles animais sob efeito de medicação analgésica a menos de 72 horas não foram considerados para fins de análise estatística.

Foram realizadas avaliações do grau de claudicação e de dor por escala analógica visual (VAS), segundo recomendações de FARIA et al. (2004) e HUDSON et al. (2004). Essas avaliações foram realizadas pelo proprietário e por um médico veterinário, de forma independente, no momento imediatamente antes da cirurgia e após sete, 15, 30, 180 e 360 dias de pós-operatório. Para avaliação da VAS foi utilizada reta desenhada sobre papel, com dez centímetros de comprimento, sendo que a extremidade à esquerda representava a ausência de dor ou claudicação (valor 0) e a extremidade à direita representava a maior dor ou a claudicação possível (valor 10). A determinação individual de cada variável, em cada momento de avaliação, foi realizada pela marcação de um ponto sobre a reta previamente determinada e posterior mensuração por régua milimetrada, fornecendo assim valor objetivo à avaliação.

O perímetro muscular da coxa foi mensurado imediatamente distal à prega inguinal por meio de fita métrica inelástica. As mensurações foram realizadas no momento imediatamente anterior à cirurgia e após sete, 15, 30, 180 e 360 dias de pós-operatório. Os ângulos máximos de flexão e extensão da articulação do quadril foram mensurados por meio de goniômetro plástico com o centro de rotação posicionado sobre o trocânter maior do fêmur. A régua fixa foi disposta sobre uma linha imaginária unindo a tuberosidade coxal à tuberosidade isquiática e a régua móvel foi posicionada sobre o eixo longo do fêmur. As mensurações foram realizadas no momento imediatamente anterior à cirurgia e após sete, 15, 30, 180 e 360 dias de pós-operatório. Para tais aferições os animais não foram sedados.

Os animais foram submetidos a exames de rotina pré-operatórios, além de outros exames necessários na avaliação individual de cada caso. Os cães tiveram uma veia cefálica cateterizada e o cateter foi conectado a equipo de soro e frasco de Ringer com lactato, com taxa de infusão aproximada de $10 \mathrm{ml} \mathrm{kg}^{-1} \mathrm{~h}^{-1}$. Posteriormente foi administrada levomepromazina ${ }^{\mathrm{a}}$ $\left(1 \mathrm{mg} \mathrm{kg}^{-1}\right)$ em associação à meperidina ${ }^{\mathrm{b}}\left(5 \mathrm{mg} \mathrm{kg}^{-1}\right)$ por via intramuscular profunda e após 15 minutos foi realizada indução anestésica com propofol ${ }^{\mathrm{C}}$ na dose média de $5 \mathrm{mg} \mathrm{kg}^{-1}$, por via intravenosa. Os animais foram então entubados e conectados a aparelho de anestesia inalatória para fornecimento de isoflurano ${ }^{\mathrm{d}}$ diluído em oxigênio para manutenção de plano anestésico compatível com o ato operatório.

Após tricotomia da região peritrocantérica, foi realizada anti-sepsia com digluconato de clorexidina 
e foi disposto o pano de campo operatório fenestrado. Com o paciente em decúbito lateral e a articulação a ser operada voltada dorsalmente, foram realizadas palpações da tuberosidade coxal, do trocânter maior e da tuberosidade isquiática, que serviram de referência para o eixo longitudinal da hemipelve e do corpo do ílio. Aproximadamente um centímetro cranial e dorsal à porção mais proximal do trocânter maior realizou-se pequena incisão cutânea com lâmina de bisturi $n^{0}$ 11, pela qual introduziu-se, a campo fechado, perpendicularmente à pele e à superfície do corpo do ílio, pino de Steinman com ponta trifacetada de 4,5mm de diâmetro, por meio dos ventres musculares glúteos, até atingir-se a superfície óssea. Posteriormente, foram realizados movimentos repetidos de raspagem do periósteo em sentido semicircular, nos sentidos cranial, craniodorsal e dorsal da borda acetabular, tomando-se cuidado para evitar a perda de contato da ponta do pino intramedular com a superfície óssea nas faces dorsal e ventral do corpo ilíaco. A ferida cutânea foi fechada por meio de adesivo cirúrgico à base de cianoacrilato ${ }^{\mathrm{e}}$. No pós-operatório, os animais receberam antiinflamatório não-esteroidal à base de carprofeno ${ }^{\mathrm{f}}$, na dose única diária de $2,2 \mathrm{mg} \mathrm{kg}^{-1}$, durante três dias (Figura 1).

Os dados paramétricos (perimetria da coxa e graus de extensão e flexão máxima do quadril) foram analisados por meio da ANOVA e do teste de Tukey para se avaliar o efeito do procedimento ao longo do período de avaliação. Os dados não-paramétricos (dor e claudicação), avaliados pelo médico veterinário e pelo proprietário, foram analisados por meio da ANOVA seguida do teste de Friedman para determinação do efeito do procedimento ao longo do tempo, de forma independente, e esses dados foram comparados entre si pelo test t de Student. Os dados foram analisados de forma independente para verificação de significância entre os cães submetidos ao procedimento de forma uni ou bilateral. Adotou-se grau de significância de 1\% $(\mathrm{P}<0,01)$.

\section{RESULTADOS E DISCUSSÃO}

Todos os animais deste estudo (48 machos e 44 fêmeas) apresentaram sinais clínicos compatíveis com dor na articulação coxofemoral e tiveram, ao exame radiográfico, diagnóstico de DAD secundária à displasia coxofemoral. A idade dos cães variou entre seis meses e oito anos, sendo que apenas nove animais tinham menos que 12 meses de idade; o peso médio foi de $31,7 \pm 8,2 \mathrm{~kg}$. A DCP foi realizada unilateralmente em 58 cães e bilateralmente em 34 animais.
A técnica da DC não apresenta restrições em relação à escolha do paciente, podendo assim ser realizada em cães de qualquer idade, o que é extremamente vantajoso em cães jovens ( $<1$ ano) e em cães idosos devido à curta duração e ao curto prazo de convalescença (KINZEL et al., 2002a,b). Essa mesma técnica pode ser realizada bilateralmente (ROCHA, 2005), sendo preservados os mesmos resultados descritos na intervenção unilateral. Tais afirmações podem ser corroboradas pela falta de diferenças estatísticas para as variáveis estudadas entre os animais submetidos à DCP uni ou bilateral. A desvantagem teórica da DC, comparada com técnicas reconstrutivas, é o fato de que só os sinais clínicos são abordados (KINZEL et al., 2002b).

Os valores obtidos em relação à dor antes da cirurgia e após sete, 15, 30, 180 e 360 dias de pósoperatório foram respectivamente de 9,78 $\pm 0,98$; $7,20 \pm 0,86 ; 1,21 \pm 0,53 ; 1,21 \pm 0,53 ; 1,21 \pm 0,53 ; 1,21 \pm 0,53$ para a avaliação realizada pelo médico veterinário e de 9,90 $\pm 1,8 ; 7,16 \pm 0,75 ; 1,01 \pm 0,03 ; 1,01 \pm 0,03 ; 1,56 \pm 0,17$ e $1,56 \pm 0,17$ para a avaliação realizada pelo proprietário. Não foram observadas diferenças significativas entre os grupos de avaliadores ao longo do período de estudo com relação aos escores de dor atribuídos pelo médico veterinário ou pelo proprietário. As médias de dor apresentaram diminuição já aos sete dias de pósoperatório, entretanto, tais diferenças só se tornaram significativas e permanentes a partir dos 15 dias de cirurgia. Não foram observadas diferenças significativas nesta variável quando considerados os animais submetidos ao procedimento uni ou bilateralmente. Apesar de os valores médios terem diminuído após a intervenção, os proprietários de quatro cães descreveram aumento da sensibilidade dolorosa após os 180 dias da intervenção cirúrgica.

KINZEL et al. (2002a) descreveram os resultados da DC em 117 cães com displasia coxofemoral e artrose, manifestando dor, e verificaram melhora clínica em 90,6\% dos cães tratados, apesar da piora inicial do quadro álgico nos primeiros três dias pós-operatórios, mas com aumento da atividade física e do fortalecimento muscular. Em estudo posterior, KINZEL et al. (2002b) descreveram os resultados a longo prazo da DC coxofemoral em 296 cães, com melhora clínica em 92\% dos casos, sendo 82\% considerados bons e $10 \%$ satisfatórios. BRAUN et al. (2003) relataram a satisfação do proprietário em 93\% de 43 cães submetidos à DC, sendo que animais com menos de um ano de idade tiveram resultados superiores àqueles mais velhos. Para FERRIGNO et al. (2004), a DC proporcionou melhora da dor já na primeira semana de pós-operatório em 91,4\% dos pacientes, tornando- 


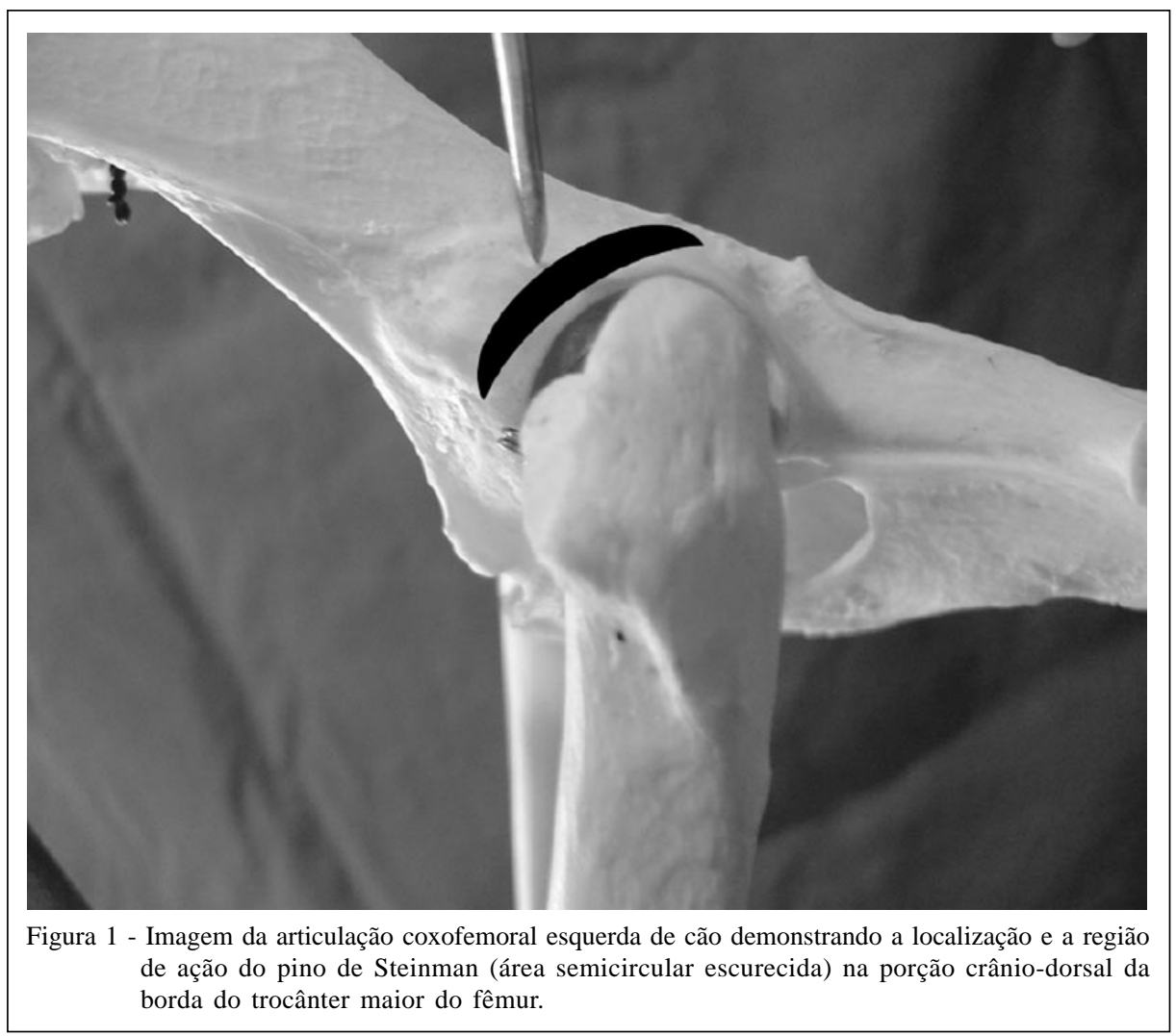

se mais evidente após o 15 dia pós-operatório. Da mesma forma, ROCHA (2005) relatou resultados satisfatórios após DC bilateral por abordagem dorsal em 10 cães, após 10 dias do procedimento. Os achados deste estudo, em que foi utilizada a DCP, vão ao encontro daqueles descritos na literatura, com melhora significativa nos escores de dor e claudicação após os 15 dias de avaliação, que se mantiveram estáveis em 95,7\% dos animais até 180 dias de pós-operatório e provavelmente são decorrentes da completa desperiostização da borda crânio-dorsal do acetábulo por via percutânea. Entretanto, especula-se que, nos quatro animais que apresentaram agravamento nas avaliações de dor e claudicação no $180^{\circ}$ dia de avaliação, a piora foi decorrente de possível reinervação da cápsula articular ou dor neuropática.

Fato relatado por $63 \%$ dos proprietários durante a primeira avaliação pós-operatória foi o aumento notável de dor nos primeiros três a cinco dias de pós-operatório sobre a região glútea, porém, este agravamento de sintomatologia não foi quantificado neste estudo. A piora do quadro de dor em momentos pós-operatórios mediatos neste estudo ocorreu provavelmente pelo trauma decorrente da inserção percutânea do pino de Steinman através da musculatura glútea, entretanto, tais achados foram transitórios, com resolução espontânea, e de duração semelhante àquele já reportado em outro estudo (KINZEL et al., 2002a). Considerando-se que os animais que apresentaram dor no período pós-operatório foram medicados com antiinflamatórios não-esteroidais e ainda assim manifestaram sensibilidade dolorosa, sugere-se a inclusão de fármaco analgésico de maior potência, como opióides, a fim de diminuir o possível desconforto resultante da DCP.

Os valores obtidos para o grau de claudicação antes da cirurgia e após sete, 15, 30, 180 e 360 dias de pós-operatório foram de 8,27 $\pm 0,7 ; 5,73 \pm 0,24$; $1,17 \pm 0,49 ; 0,28 \pm 0,03 ; 0,00 \pm 0,00 ; 0,00 \pm 0,00$ e $9,57 \pm 1,4$; $4,26 \pm 0,91 ; 2,01 \pm 0,84 ; 0,61 \pm 0,01 ; 0,04 \pm 0,01 ; 0,06 \pm 0,01$, para as avaliações do médico veterinário e dos proprietários, respectivamente. O grau de claudicação apresentou comportamento similar ao da dor, sendo que já aos sete dias de pós-operatório observou-se diminuição nos escores de claudicação atribuídos pelo médico veterinário e pelos proprietários, sendo que diferenças significativas foram observadas a partir de 15 dias de pós-operatório. Não foram observadas diferenças entre os escores atribuídos pelos dois grupos de avaliadores, tampouco houve diferenças entre os animais submetidos à DCP uni ou bilateral. 
KINZEL et al. (2002b), utilizando-se da técnica de DC a céu aberto em 117 cães, avaliaram o grau de claudicação, dor e massa muscular por meio de escala numérica ordinal e demonstraram que 50,4\% dos animais já se apresentavam livres da sintomatologia atribuída à DCF aos dois meses do pós-operatório, enquanto $28,2 \%$ dos animais apresentavam sinais insignificantes. De acordo com aqueles autores, em $90,6 \%$ dos animais que apresentavam os sinais mais evidentes e severos, 78,6\% obtiveram ótimos resultados, apresentando melhora da claudicação, ausência de dor na extensão do quadril e recuperação da musculatura envolvida. ROCHA (2005) observou melhora discreta em $60 \%$ dos animais operados após sete dias e destacou ainda que, apesar da melhora, esta foi discreta, apresentando limitada redução de dor, sendo compatível com os resultados revisados na literatura (KINZEL et al., 2002b; FERRIGNO et al., 2004). Tais resultados indicam que essa limitação foi justificada, no caso da DC aberta, pelo fato de que no local cirúrgico, assim como nos tecidos adjacentes, a reatividade aos estímulos mecânicos fica aumentada em razão da ação de mediadores inflamatórios e essa sensibilização piora com a ação mecânica da cabeça do fêmur instável. Apesar disso, houve melhora significativa da dor aos 15 dias de pós-operatório, e aos 30 dias os mesmos resultados foram mantidos. Aos 30 dias de pós-operatório, na avaliação de ROCHA (2005), os animais apresentaram 90\% de melhora na dor. Para FERRIGNO et al. (2006), aos 30 dias de pósoperatório, 100\% dos animais de um total de 67 cães operados apresentavam-se com dor mínima e insignificante. Aos 180 dias de pós-operatório, os autores destacaram que $95,8 \%$ dos animais apresentavam o mesmo resultado e aos 360 dias de pós-operatório 94,1\% apresentaram o mesmo escore. Apesar de KINZEL et al. (2002a) recomendarem a inclusão das inserções dos músculos reto femoral e glúteo profundo na desperiostização acetabular, neste estudo, por via percutânea, não foi possível verificar esta manobra. No entanto, os resultados clínicos após o procedimento minimamente invasivo apresentou similitude àqueles já descritos na literatura. Dessa forma, estudos anatômicos e clínicos são necessários para determinar o real efeito da inclusão das inserções musculares no alívio da dor.

A melhora dos valores determinados em relação à dor e à claudicação nos animais deste estudo, submetidos à DCP, corresponde à melhora clínica em quase $96 \%$ dos animais. Tais observações não podem ser comparadas diretamente pelas diferenças inerentes nas escalas de avaliação entre os estudos, não obstante, indicam que a DCP promove resultados similares àqueles obtidos pela DC. Apesar da melhora evidente a partir dos 180 dias de pós-operatório na grande maioria dos estudos, alguns animais apresentaram piora do quadro de dor, o que também foi observado no estudo de ROCHA (2005), que relatou recidiva da dor em $10 \%$ dos pacientes, e também por BRAUN et al. (2003), que observaram 32,6\% de recidiva da dor em um período de 3,5 anos de avaliação. Tal fato deve-se à possível reinervação da cápsula articular coxofemoral ou pode estar atribuído a alguma outra doença que começa a se manifestar após a cirurgia. No estudo de KINZEL et al. (2002a), em que a DC foi avaliada por um período de 10 anos de pós-operatório, os autores citam que uma possível reinervação não pôde ser comprovada, pois os cães apresentavam-se livre de dores.

Em relação a observações sobre o volume da massa muscular, somente o estudo de ROCHA(2005) menciona dados sobre esta variável e, embora o autor tenha descrito aumento da mesma após a DC, não levou em consideração que $60 \%$ dos animais em seu estudo eram jovens com menos de um ano de idade e conseqüentemente a melhora observada pode ter sido resultante do próprio ganho de massa muscular inerente ao crescimento de cada indivíduo. Neste estudo, os valores médios de perimetria da coxa apresentaram discreta diminuição aos sete e aos 15 dias de pósoperatório em relação ao momento pré-operatório. No entanto, aos 30 dias de avaliação, pôde-se notar aumento significativo destes valores de forma progressiva até o término deste estudo (T0 = $38,3 \pm 4,0 \mathrm{~cm} ; \mathrm{T} 7=38,2 \pm 3,9 \mathrm{~cm} ; \mathrm{T} 15=38,2 \pm 4,0 \mathrm{~cm} ; \mathrm{T} 30=$ $38,8 \pm 4,1 \mathrm{~cm} ; \mathrm{T} 180=39,7 \pm 4,2 \mathrm{~cm} \mathrm{e} \mathrm{T360}=40,3 \pm 3,8 \mathrm{~cm}$ ), mesmo quando os nove animais em fase de crescimento foram excluídos da análise. Dessa forma, acredita-se que o ganho de massa muscular foi decorrente da diminuição do grau de dor e conseqüentemente da claudicação do aumento do grau de amplitude de movimento articular, conforme observado pelo aumento significativo dos valores de extensão máxima passiva após 15 dias de pós-operatório com aumento gradual nesta variável até o término do estudo ( $\mathrm{T} 0=132,7 \pm 5,5^{\circ}$; $\mathrm{T} 7=134,4 \pm 5,3^{\circ} ; \mathrm{T} 15=137,8 \pm 5,6^{\circ} ; \mathrm{T} 30=142,6 \pm 5,9^{\circ}$; $\mathrm{T} 180=147,3 \pm 6,4^{\circ}$ e T $\left.360=147,0 \pm 5,8^{\circ}\right)$. Em contrapartida, os valores médios de flexão articular passiva (T0 = $50,0 \pm 1,8^{\circ} ; \mathrm{T} 7=49,3 \pm 1,6^{\circ} ; \mathrm{T} 15=49,0 \pm 1,3^{\circ} ; \mathrm{T} 30=$ $48,8 \pm 1,3^{\circ} ; \mathrm{T} 180=48,7 \pm 1,1^{\circ}$ e T360 $=48,7 \pm 1,1^{\circ}$ ), apesar de apresentarem decréscimo, o que significou maior capacidade de flexão, não sofreram alterações significativas ao longo do período de estudo. Apesar da pouca importância dada à goniometria na maioria dos estudos consultados, este dado representa avaliação simples e objetiva da evolução pós-operatória (JAEGGER et al., 2002). 
O sucesso da DC como método cirúrgico para o tratamento da DCF depende da destruição direcionada das vias nervosas sensíveis, as quais suprem as cápsulas das articulações coxofemorais (KINZEL et al., 1998). No entanto, para a realização do procedimento, é necessário conhecer a anatomia topográfica exata do suprimento nervoso da articulação coxofemoral (KINZEL et al., 1998, SCHMAEDECKE, 2004). A abordagem tradicional à articulação coxofemoral permite adequada exposição da borda cranial da articulação e limitada visibilidade da borda acetabular dorsal (KINZEL et al., 2002 a,b; FERRIGNO et al., 2007),enquanto que a abordagem percutânea não permite visibilidade das estruturas periarticulares de interesse. A proximidade do nervo glúteo cranial, da artéria e da veia glútea ao sítio de penetração do pino de Steinman utilizado na DCP poderia predispor lesões destas estruturas, entretanto, não foram observadas complicações que pudessem ser atribuídas à abordagem percutânea. Na opinião dos autores, a introdução do pino de Steinman perpendicular à superfície da pele e na face crânio-dorsal do trocânter maior evitaria complicações aos nervos e aos vasos glúteos, enquanto que a manutenção do contato do pino intramedular com a cortical óssea periacetabular durante a DCP evitaria lesões ao nervo ciático.

\section{CONCLUSÃO}

A partir dos resultados obtidos, conclui-se que a DCP propicia alívio da sintomatologia clínica com conseqüente aumento da extensão articular passiva e da massa muscular da coxa, a despeito do incremento do quadro álgico no período pós-operatório mediato.

\section{FONTES DE AQUISIÇÃO}

\author{
aNeozine. Aventis Pharma, Suzano/SP. \\ bDolosal. Cristália, Itapira/SP. \\ 'Provive. Claris, São Paulo/SP. \\ ${ }^{\mathrm{d} I s o f o r i n e . ~ C r i s t a ́ l i a, ~ I t a p i r a / S P . ~}$ \\ 'Vetbond. 3M, Campinas/SP.

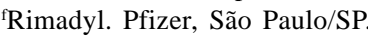

\section{REFERÊNCIAS}

BRAUN, D. et al. Dorsale denervation der hüftgelenkskapsel beim hund ergebnisse einer langzeitstudie. Kleintierpraxis, Hannover, v.4, p.185-244, 2003.

DASSLER, D.L. Canine hip dysplasia: diagnosis and nonsurgical treatment. In: SLATTER, D. Textbook of small animal surgery. 3.ed. Philadelphia: Saunders, 2003. V.2, p.20192029 .
DUELAND, R.T. et al. Effects of pubic symphysiodesis in dysplastic puppies. Veterinary Surgery, Boston, v.30, n.3, p.201-217, 2001.

FARIA, M.L.E. et al. Comparison between visual analogue scale and a numerical rating scale in lameness observation in dogs. Veterinary Surgery, Boston, v.33, p.E-8, 2004.

FERRIGNO, C.R.A. et al. Estudo da técnica de denervação da cápsula articular coxofemoral no tratamento da dor em cães com displasia coxofemoral: resultados preliminares. In: CONGRESSO DO COLÉGIO BRASILEIRO DE CIRURGIA E ANESTESIOLOGIA VETERINÁRIA, 6., 2004, São Paulo. Anais... São Paulo, CBCAV, 2004. p.169-170.

FERRIGNO, C.R.A. et al. Denervação acetabular dorsal em cães - 360 dias de avaliação. In: CONGRESSO DO COLÉGIO BRASILEIRO DE CIRURGIA E ANESTESIOLOGIA VETERINÁRIA, 7, 2006, Belo Horizonte. Arquivo Brasileiro de Medicina Veterinária e Zootecnia, Belo Horizonte, v.58, supl.2, p.21, 2006.

FERRIGNO, C.R.A. et al. Abordagem cirúrgica inédita para denervação acetabular em cães. Pesquisa Veterinária Brasileira, Seropédica. v.27, n.2, p.61-63, 2007.

GASSE, H. et al. Zur innervation der hüftgelenkkapsel beim hund. Kleintierpraxis, Hannover, v.41, p.883-886, 1996.

HUDSON, J.T. et al. Assessing repeatability and validity of a visual analogue scale questionnaire for use in assessing pain and lameness in dogs. American Journal of Veterinary Research, Schaumburg, v.65, n.12, p.1634-1643, 2004.

HULSE, D.A.; JOHNSON, A.L. Tratamento da doença articular. In: FOSSUM, T.W. Cirurgia de pequenos animais. São Paulo: Roca, 2002. p.1042-1050.

JAEGGER, G. et al. Reliability of goniometry in labrador retrievers. American Journal of Veterinary Research, Schaumburg, v.63, n.7, p.979-986, 2002.

KINZEL, S. et al. Sensory innervation of the hip joint capsule in dogs. Tierarztliche Praxis, Berlim, v.26, n.5, p.330-335, 1998.

KINZEL, S. et al. 10 years experience with denervation of the hip joint capsule for treatment of canine hip joint dysplasia and arthrosis. Berliner Munchener Tierarztliche Wochenschrifft, Berlim, v.115, n.1-2, p.53-56, 2002a.

KINZEL, S. et al. Clinical evaluation of denervation of the canine hip joint capsule: a retrospective study of 117 dogs. Veterinary Comparative Orthopaedics and Traumatology, Stuttgart, v.15, p.51-56, 2002 b.

OLMSTEAD, M.L. et al. A five year study of 221 total hip replacements in the dog. Journal of the American Veterinary Medical Association, Schaumburg, v.183, n.2, p.191-194, 1983.

RAWSON, E.A. et al. Simultaneous bilateral femoral head and neck ostectomy for the treatment of canine hip dysplasia. 
Journal of the American Animal Hospital Association, Denver, v.41, p.166-170, 2005.

ROCHA, L.B. Denervação articular em cães com osteoartrose coxofemoral secundária a displasia. 2005. 85f. Dissertação (Mestrado em Ciência Veterinária) - Programa de Pós-graduação em Ciência Veterinária, Universidade Federal Rural de Pernambuco, Recife.

SCHMAEDECKE, A. Estudo quantitativo das fibras nervosas do periósteo acetabular em cães. 2004. 103f.

Dissertação (Mestrado em Anatomia dos Animais Domésticos)
- Programa de Pós-graduação em Anatomia dos animais domésticos e silvestres, Universidade de São Paulo.

SCHULZ, K.S.; DEJARDIN, L.M. Surgical treatment of canine hip dysplasia. In: SLATTER, D. Textbook of small animal surgery. 3.ed. Philadelphia: Saunders, 2003. Cap.145, p.20292059.

TOMLINSON, J.; McLAUGHLIN, R. Medically managing canine hip dysplasia. Veterinary Medicine, v.91, n.1, p.4853, 1996 . 